\title{
Generalized Clifford parallelisms
}

\author{
Andrea Blunck Stefano Pasotti Silvia Pianta*
}

Dedicated to Mario Marchi on the occasion of his 70th birthday

\begin{abstract}
We define generalized Clifford parallelisms in $\mathrm{PG}(3, F)$ with the help of a quaternion skew field $H$ over a field $F$ of arbitrary characteristic. Moreover we give a geometric description of such parallelisms involving hyperbolic quadrics in projective spaces over suitable quadratic extensions of $F$.
\end{abstract}

Keywords: Clifford parallelism, quadratic forms, quadrics, quaternions

MSC 2000: 51A15, 51J15, 11E04

\section{Introduction}

It is known that the three dimensional real projective space $\operatorname{PG}(3, \mathbb{R})$ can be endowed with two projectively equivalent parallelisms, namely the left and right Clifford parallelisms, related to left and right multiplications in the Hamilton quaternion algebra $\mathbb{H}(\mathbb{R})$ (see e.g. [15]). For these parallelisms there are many equivalent geometric representations (see e.g. [22, Sec. 142], [8, 12 A], [16, Chapter 14]). In particular each parallel class can be described considering the lines that meet a fixed imaginary line (and its conjugate) belonging to one of the two reguli of a complex hyperbolic quadric whose points do not belong to $\mathrm{PG}(3, \mathbb{R})$.

The aim of this work is to extend these notions to the projective 3-space over a general (commutative) field of arbitrary characteristic. This can be done in several ways, using constructions that involve either rings of generalized quaternions, or the notions of Baer subspace of a projective space and indicator set of

\footnotetext{
${ }^{*}$ Research supported by MIUR (Italy), GNSAGA of INdAM (Italy) and Fondazione Giuseppe Tovini of Brescia (Italy)
} 





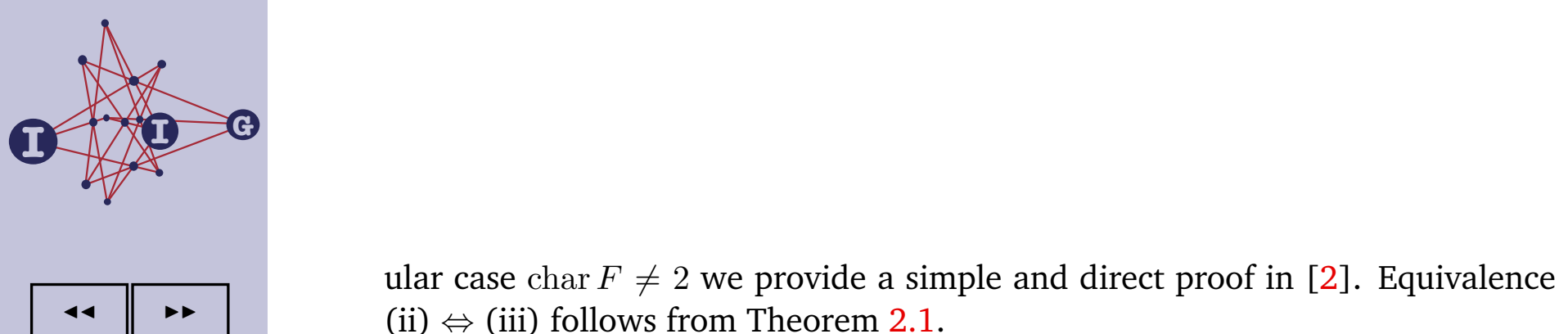
(ii) $\Leftrightarrow$ (iii) follows from Theorem 2.1 .

2.3 Proposition. Let $q$ be a 4-dimensional regular quadratic form over a field $F$. If $d(q)$ is trivial, then $q$ is similar to the norm form of a suitable quaternion algebra $H$ over $F$, and $H$ is a skew field if and only if $q$ is anisotropic over $F$. If $d(q)$ is nontrivial, then there exists a quadratic extension $F^{\prime}$ of $F$ (namely the discriminant extension) such that $q_{F^{\prime}}$ is similar to the norm form of a quaternion algebra $H$ over $F^{\prime}$, and $H$ is a skew field if and only if $q_{F^{\prime}}$ is anisotropic over $F^{\prime}$. Conversely if $q$ is isometric to the norm form of a quaternion algebra $H$ over a field $F$, then $d(q)$ is trivial.

Proof. Assume $d(q)$ is trivial. Then by [6, Lemma 4.4] $q$ is either hyperbolic or similar to the orthogonal sum of quadratic forms $s_{1} N \perp s_{2} N$ for suitable $s_{1}, s_{2} \in F^{*}$, where $N$ is the norm of a separable quadratic extension $K / F$. In the first case $q$ is similar to the norm form of a split quaternion algebra, and this happens exactly when $q$ is isotropic over $F$. If, on the contrary, $q$ is anisotropic over $F$, then $q$ is similar to the norm form of the quaternion algebra $H=\left(K / F, s_{1}^{-1} s_{2}\right)$. If $d(q)$ is non-trivial and $d$ is a representative for the square class of $d(q)$, the same as above holds over the discriminant quadratic extension $F^{\prime}=F(\sqrt{d})$ of $F$. The converse is obvious.

2.4 Proposition. Let $\mathcal{Q}$ be a quadric of $\mathrm{PG}(3, F)$ and write $q$ for a representative of the similarity class of quadratic forms associated to $\mathcal{Q}$ in the vector space $F^{4}$. Then the following hold:

(i) $\mathcal{Q}$ is hyperbolic if and only if $q$ is isometric to the norm form of the split quaternion algebra $\mathrm{M}_{2}(F)$ over $F$.

(ii) $\mathcal{Q}$ has no points in $\mathrm{PG}(3, F)$ and there exists a separable quadratic extension $K$ of $F$ such that $\mathcal{Q}_{K}$ is hyperbolic in $\mathrm{PG}(3, K)$ if and only if $q$ is isometric to the norm form of a quaternion skew field $H$ over $F$.

Proof. (i) is obvious. To prove claim (ii) note that, if $q$ is anisotropic and $q_{K}$ hyperbolic, by [6, Lemma 4.2], $q$ is similar to the quadratic form $s_{1} N \perp s_{2} N$, where $N$ is the norm of the extension $K / F$, and thus, as in the proof of the previous proposition, $q$ is similar to the norm form of a suitable quaternion algebra $H$. Since $q$ is anisotropic over $F, H$ is a division algebra.

Conversely if $q$ is isometric to the norm form of a quaternion skew field $H$ over $F$, then $\mathcal{Q}$ does not have points in $\mathrm{PG}(3, F)$ by Theorem 2.1. Moreover $H$ contains a maximal separable subfield $K$, so $q_{K}$ is hyperbolic by Theorem 2.2 and $\mathcal{Q}_{K}$ is then hyperbolic in $\mathrm{PG}(3, K)$. 





Proof. We distinguish the cases $L=K=F+F i$ and $L=K^{\prime}=F+F j$. First consider the line $L=K$. Each line of the right parallel class of $K$ has the form $K h$ for some $h \in H^{*}$, thus it is spanned by $h=\left(\begin{array}{cc}x & y \\ b \bar{y} & \bar{x}\end{array}\right)$ and $i h=\left(\begin{array}{cc}i x & i y \\ b \bar{i} \bar{y} & \bar{i} \bar{x}\end{array}\right)$. The points of intersection of $K h$ and $\mathcal{Q}_{K}$ are exactly the points $p=K M$, where $M$ is a non-invertible $K$-linear combination of these two matrices. One obtains the two solutions

$$
\begin{aligned}
p & =K M, \quad \text { with } M=-\bar{i}\left(\begin{array}{cc}
x & y \\
b \bar{y} & \bar{x}
\end{array}\right)+\left(\begin{array}{cc}
i x & i y \\
b \bar{i} \bar{y} & \bar{i} \bar{x}
\end{array}\right)=(i-\bar{i})\left(\begin{array}{cc}
x & y \\
0 & 0
\end{array}\right) \text { and } \\
p^{\prime} & =K M^{\prime}, \text { with } M^{\prime}=-i\left(\begin{array}{cc}
x & y \\
b \bar{y} & \bar{x}
\end{array}\right)+\left(\begin{array}{cc}
i x & i y \\
b \bar{i} \bar{y} & \bar{i} \bar{x}
\end{array}\right)=(\bar{i}-i)\left(\begin{array}{cc}
0 & 0 \\
b \bar{y} & \bar{x}
\end{array}\right) .
\end{aligned}
$$

Obviously (cfr. Proposition 4.1), the matrices $M$ from above, with $x, y \in K$, are exactly the elements of $I=I_{U} \in \mathcal{R}_{K}$, where $U=K(0,1)$. In particular, the right parallel class of $K$ is indicated by $I$. Similarly, one can show that the left parallel class of $K$ is indicated by $J=I^{V} \in\left(\mathcal{R}_{K}\right)_{\text {opp }}$, where $V=K(1,0)$.

Assume now char $F=2$ and consider the inseparable extension $L=K^{\prime}=$ $F+F j$. Again each line of the right parallel class of $K^{\prime}$ is spanned by $h$ and $j h$ for a suitable $h \in H^{*}$. We consider $H$ as a subring of $H_{K^{\prime}}$ (cfr. Lemma 4.3(ii)), and thus $h=\left(\begin{array}{ll}x & y \\ z & t\end{array}\right)$ and $j h=\left(\begin{array}{ll}j z & j t \\ j x & j y\end{array}\right)$. The points of intersection of $K^{\prime} h$ and $\mathcal{Q}_{K^{\prime}}$ are the points $p=K^{\prime} M$ where $M$ is a non-invertible $K^{\prime}$-linear combination of $h$ and $j h$. A straightforward computation shows that, assuming as a consequence of $h \in H^{*}$ that $\operatorname{det}(h) \neq 0$, the only solution is

$$
p=K^{\prime} M, \text { with } M=j\left(\begin{array}{ll}
x+z & y+t \\
x+z & y+t
\end{array}\right)
$$

and, again by Proposition 4.1, the matrices $M$ of this form are the elements of $I=I_{U} \in \mathcal{R}_{K^{\prime}}$, where $U=K^{\prime}(1,1)$. Similarly one can show that the left parallel class of $K^{\prime}$ is indicated by $J=I^{U} \in\left(\mathcal{R}_{K^{\prime}}\right)_{\mathrm{opp}}$.

In the remainder of this proof there is no need to distinguish any more the separable case from the inseparable one, thus, from now on, writing $L$ we mean either $K$ or $K^{\prime}$. We consider now $R=c^{-1} L c, c \in H^{*}$. Let $\alpha$ be the collineation of PG $(3, L)$ induced by the conjugation $x \mapsto c^{-1} x c$. Then $\alpha$ leaves both PG $(3, F)$ and $\mathcal{Q}_{L}$ invariant. Moreover,

$$
u \in \operatorname{ker} M \Longleftrightarrow u c \in \operatorname{ker} c^{-1} M c, \quad u \in \operatorname{im} M \Longleftrightarrow u c \in \operatorname{im} c^{-1} M c
$$

implies that $\alpha$ leaves $\mathcal{R}_{L}$ and $\left(\mathcal{R}_{L}\right)_{\text {opp }}$ invariant. Since $\alpha$ maps the right (left) parallel class of $L$ to the right (left) parallel class of $R$ we conclude that the right (left) parallel class of $R$ is indicated by $I^{\alpha} \in \mathcal{R}_{L}$ (or $J^{\alpha} \in\left(\mathcal{R}_{L}\right)_{\text {opp }}$, respectively). 
It remains to show that all spreads indicated by a line of $\mathcal{R}_{L}$ (or $\left(\mathcal{R}_{L}\right)_{\text {opp }}$ ) are right (left) parallel classes of lines $c^{-1} L c$. But this follows from the above by (4.1), since for each 1-dimensional subspace $W \leq L^{2}$ there is a $c \in H^{*}$ with $W=U c$ (or $W=V c$, respectively).

Note that, in the proof above, in the separable case we have $p^{\prime}=p^{\tilde{\kappa}}$ (see Remark 4.4). So the right parallel class of $K$ is also indicated by $I^{\prime}=I^{\tilde{\kappa}}$.

Since each (right or left) parallel class has a representative through $\mathbf{1}$, which is a maximal commutative subfield of $H$, by Proposition 4.2 we can describe it as $\mathcal{S}(I)$, with $I$ a line in an appropriate Baer superspace of $\operatorname{PG}(3, F)$. In particular, we get the following.

4.8 Corollary. The right and left Clifford parallelisms are regular.

In the special case that $F$ admits only one quadratic extension $K$ (and hence $K / F$ is separable, thus char $F \neq 2$ ), the Clifford parallelisms are indicated by exactly the lines of a regulus and its opposite in $\mathrm{PG}(3, K)$. For $F=\mathbb{R}$ and $K=\mathbb{C}$ this is well known, see e.g. [8, 12 A]. This observation leads to the following corollary.

4.9 Corollary. Let $F, K$ and $H$ be as before. Then all quadratic extensions of $F$ in $H$ are conjugate to $K$ if and only if there exists a hyperbolic quadric $\mathcal{Q}$ in $\mathrm{PG}(3, K)$ having no points in $\mathrm{PG}(3, F)$ and incident with every line of $\mathrm{PG}(3, F)$.

In general, however, we need more than one Baer superspace $\mathrm{PG}(3, L)$. In order to get a unified description of the entire parallelisms, we proceed as follows: Let $\widehat{F}$ be the quadratic closure of $F$. Then all quadratic extensions $L$ of $F$ are contained in $\widehat{F}$. We consider $\mathrm{PG}(3, F)$ and all $\mathrm{PG}(3, L)$ as subspaces of $\mathrm{PG}(3, \widehat{F})$. More explicitly, we take $H$ as underlying vector space of $\mathrm{PG}(3, F)$ and $H_{L}$ or $H_{\widehat{F}}$ (with the same basis $(1, i, j, i j)$ ) as underlying vector spaces of $\mathrm{PG}(3, L)$ or $\mathrm{PG}(3, \widehat{F})$, respectively. In particular, for any two distinct separable quadratic extensions $L, L^{\prime}$ we have $\mathrm{PG}(3, L) \cap \mathrm{PG}\left(3, L^{\prime}\right)=\mathrm{PG}(3, F)$.

In addition, we consider in $\mathrm{PG}(3, \widehat{F})$ and in all $\mathrm{PG}(3, L)$ the quadrics $\mathcal{Q}_{\widehat{F}}$ and $\mathcal{Q}_{L}$ associated to the norm of $H$. By Theorem 2.2 the quadric $\mathcal{Q}_{L}$ is empty exactly if $L$ is not a subalgebra of $H$, and hyperbolic otherwise. This implies that also $\mathcal{Q}_{\widehat{F}}$ is hyperbolic. Let the reguli $\mathcal{R}_{\widehat{F}}$ on $\mathcal{Q}_{\widehat{F}}$ and $\mathcal{R}_{L}$ on $\mathcal{Q}_{L}$ be defined as in Proposition 4.1. Then $\mathcal{R}_{L}$ consists exactly of those lines of $\mathcal{R}_{\widehat{F}}$ that belong to $\mathrm{PG}(3, L)$, the same holds for the opposite reguli. In case that $\mathcal{Q}_{L}=\emptyset$ we set $\mathcal{R}_{L}:=\emptyset=:\left(\mathcal{R}_{L}\right)_{\text {opp }}$.

For a line $I$ on $\mathcal{Q}_{\widehat{F}}$ we can define $\mathcal{S}(I)$ only if $I$ belongs to some $\mathrm{PG}(3, L)$; note that of course we then only consider the points of $I$ that are points of $\mathrm{PG}(3, L)$. 


[6] T. De Medts, A characterization of quadratic forms of type $E_{6}, E_{7}$ and $E_{8}, J$. Algebra 252 (2002), 394-410.

[7] P. K. Draxl, Skew Fields, London Math. Soc. Lecture Note Ser. 81, Cambridge Univ. Press, Cambridge, 1983.

[8] O. Giering, Vorlesungen über höhere Geometrie, Vieweg, Braunschweig, 1982.

[9] T. Grundhöfer, Reguli in Faserungen projektiver Räume, Geom. Dedicata 11 (1981), 227-237.

[10] A. Hahn, Quadratic Algebras, Clifford Algebras and Arithmetic Witt Groups, Springer-Verlag, Berlin, 1994.

[11] H. Havlicek, Spreads of right quadratic skew field extensions, Geom. Dedicata 49 (1994), 239-251.

[12] , On Plücker transformations of generalized elliptic spaces, Rend. Mat. Appl. 15 (1995), 39-56.

[13] A characteristic property of elliptic Plücker transformations, $J$. Geom. 58 (1997), 106-116.

[14] H. Havlicek and S. Pasotti, A survey on the notion of regulus in a skew space, Quad. Sem. Mat. Brescia (2003), 1-32.

[15] H. Karzel, Kinematic spaces, in Symposia Mathematica, Vol. XI (Convegno di Geometria, INDAM, Rome, 1972), Academic Press, London, 1973, pp. 413-439.

[16] H. Karzel and H.-J. Kroll, Geschichte der Geometrie seit Hilbert, Wiss. Buchges., Darmstadt, 1988.

[17] N. Knarr, Translation Planes. Foundations and construction principles, Lecture Notes in Math. 1611, Springer-Verlag, Berlin, 1995.

[18] T. Y. Lam, The Algebraic Theory of Quadratic Forms, Math. Lecture Note Ser., W. A. Benjamin, Inc., Reading, Mass., 1973.

[19] W. Scharlau, Quadratic and Hermitian Forms, Grundlehren Math. Wiss. 270, Springer-Verlag, Berlin, 1985.

[20] B. Segre, Lectures on Modern Geometry. With an appendix by Lucio Lombardo-Radice, Edizioni Cremonese, Rome, 1961.

[21] J. Tits and R. Weiss, Moufang Polygons, Springer Monogr. Math., SpringerVerlag, Berlin, 2002. 
[22] O. Veblen and J. Young, Projective Geometry, vol. II, Ginn and Company, Boston, 1918.

[23] M.-F. Vignéras, Arithmétique des Algèbres de Quaternions, Lecture Notes in Math. 800, Springer, Berlin, 1980.

go back

full screen

close

quit

Andrea Blunck

Dept. of Mathematics, Universität Hamburg, Bundesstr. 55, D-20146 Hamburg

e-mail: andrea.blunck@math.uni-hamburg.de

website: http://www.math.uni-hamburg.de/home/blunck/

Stefano Pasotti

Dept. of Mathematics, Università degli Studi di Brescia, via Valotti, 9, I-25133 Brescia

e-mail: stefano.pasotti@ing.unibs.it

website: http://www.ing.unibs.it/〜stefano.pasotti

Silvia Pianta

Dept. of Mathematics, Università Cattolica del Sacro Cuore, via Trieste, 17, I-25121 BRESCIA

e-mail: pianta@dmf.unicatt.it 